\title{
Complications of electrotherapy - the dark side of treatment with cardiac implantable electronic devices
}

\author{
Szymon Domagała ${ }^{1}$, Michał Domagała $^{2}$, Jakub Chyła ${ }^{3}$, Celina Wojciechowska ${ }^{4}$, Marianna Janion ${ }^{1,5}$, \\ Anna Polewczyk ${ }^{1,5}$ \\ ${ }^{1} 2^{\text {nd }}$ Department of Cardiology, Swietokrzyskie Cardiology Center, Kielce, Poland \\ ${ }^{2}$ Department of Anaesthesiology and Intensive Care, St. Luke's Hospital, Końskie, Poland \\ ${ }^{3}$ Faculty of Medicine, Jagiellonian University Medical College, Krakow, Poland \\ ${ }^{4} 2^{\text {nd }}$ Department of Cardiology, Medical University of Silesia in Katowice, School of Medicine with the Division of Dentistry in Zabrze, Poland \\ ${ }^{5}$ Department of Internal Diseases, Cardiology and Medicine Nursing, Institute of Nursing and Obstetrics, Faculty of Medicine and Health \\ Sciences, Jan Kochanowski University, Kielce, Poland
}

Adv Interv Cardiol 2018; 14, 1 (51): 15-25

DOI: https://doi.org/10.5114/aic.2018.74351

\begin{abstract}
A bstract
In the past years we have been observing the dynamic development of electrotherapy, as evidenced by the steadily rising number of implanted pacemakers (PM), as well as devices used in the treatment of dangerous arrhythmia and heart failure, such as implantable cardioverter defibrillators (ICD) and cardiac resynchronisation therapy (CRT-P/D). This is a consequence of the ageing of the populations of the majority of developed countries and also the gradually widening indications for the use of such devices. Along with the observed rise in the number of new implantations, the number of complications of electrotherapy is rising as well. In view of the increase in the incidence of complications, it is necessary to systematize the knowledge on this subject, because there is still no official classification of this type of complication and guidelines for dealing with such cases do not appear to cover the scale of the problem. In addition, late complications of electrotherapy play the most important role, in which case the removal of the entire pacing system, transvenous lead extraction (TLE), is a challenge due to the older age of leads strongly attached to the venous walls and endocardium of the heart cavity. The present paper presents a modern classification of electrotherapy complications and discusses the types of complications according to the most recent literature reports. Moreover, the diagnosis and management of particular types of complications with the assessment of indications for TLE are discussed.
\end{abstract}

Key words: electrotherapy complications, lead-related endocarditis, dysfunctions of the leads, transvenous lead extraction.

\section{Introduction}

Electrotherapy complications, although thoroughly neglected by history, have accompanied the implantation of cardiac pacing systems since the very beginning of this type of surgery. The cardiac pacemaker, first implanted by Ake Senning in Stockholm in 1958, worked for only a few hours because of the operator's failure to stimulate the pacemaker [1]. The first pacemaker implanted in Poland, in Gdansk in 1963, stopped functioning after a few months due to spontaneous damage to the device casing and its destruction by tissue fluids [2]. Although malfunctions of the electronic systems of the device are rare today, the number of complications associated with the presence of the leads is constantly increasing $[3,4]$.

\section{Classification of electrotherapy complications}

The lack of a uniform classification of complications and heterogeneous terminology contribute to the problem of establishing a treatment regimen in patients with complications associated with PM/ICD/CRT implantation. The most clinically relevant view seems to be the one proposed by Kutarski (unpublished) presented in Table I. The classification was made taking into account the etiology of complications and their clinical relevance.

\section{The criterion of time}

The generally accepted classification, taking into account the criterion of time, divides the complications into 
Table I. Classification of complications

\begin{tabular}{|c|c|}
\hline \multirow{8}{*}{$\begin{array}{l}\text { Early } \\
\text { complications }\end{array}$} & Dislodgement of the lead \\
\hline & $\begin{array}{l}\text { Improper location of the lead (left atrium, } \\
\text { left ventricle, aorta, pulmonary trunk) }\end{array}$ \\
\hline & Early perforation \\
\hline & Pocket hematoma \\
\hline & Pneumothorax (and pleural hematoma) \\
\hline & Venous thrombosis \\
\hline & Early dysfunction of the lead \\
\hline & Early postoperative infection (local and sepsis) \\
\hline \multirow[t]{10}{*}{$\begin{array}{l}\text { Late } \\
\text { complications }\end{array}$} & $\begin{array}{l}\text { Decubitus of the pocket and threatening } \\
\text { decubitus }\end{array}$ \\
\hline & Chronic infection of the pocket unit \\
\hline & $\begin{array}{l}\text { Lead-related infective endocarditis (all forms } \\
\text { of generalized infection) }\end{array}$ \\
\hline & $\begin{array}{l}\text { Late lead dysfunction (without exit and entry } \\
\text { block) }\end{array}$ \\
\hline & Late perforation \\
\hline & Late recognized old lead dislocation \\
\hline & Lead breaking and lead migration \\
\hline & Late recognized improper lead location \\
\hline & Lead-related venous occlusion \\
\hline & Lead-related tricuspid valve dysfunction \\
\hline
\end{tabular}

early and late. The problem is the lack of consistency in the time that would be the dividing line. In the literature, the limit for early complications is 1 month after the procedure [3] but also 1 year as in the case of infectious complications associated with artificial valves [5, 6]. The second criterion mentioned above is that the lead is usually removed by simple traction by this time, and after more than 1 year, due to the healing and ingrowing process, it is often necessary to use more complex equipment to perform transvenous lead extraction (TLE) [7].

\section{Early complications}

Complications related to improper location of leads and lead dislodgement

Within the early complications, a large group is associated with improper location of the leads, which may be initially incorrect or may be a consequence of an unintentional translocation during the postoperative period. Inadvertent implantation of the leads to the left chambers of the heart is very rare $(<1 \%$ procedures) [8]. There have been reports of unplanned left atrial or left ventricular implantation as a result of the lead passing through the patent foramen ovale or atrial/interventricular septal defect [4, 9] (Figures 1 A, B). Another reason may be the insertion of the lead directly into the left ventricle or aorta through the arterial system [10-12]. The position of the leads in the wide range of arterial systems may lead to serious consequences for the patient, who is exposed to thromboembolic events such as systemic embolism or stroke [9, 12, 13]. Due to the high probability of these complications and their severity, these patients are the most likely to be qualified for TLE $[13,14]$. In cases where this is not possible for whatever reason, chronic anticoagulant therapy should be implemented [10, 14]. However, the key to preventing such complications is the early detection of improper locations of the leads by fluoroscopy and ECG tracing during the procedure or classic $X$-ray picture of the chest and echocardiography after the procedure [15]. Other possible improper locations include the superior vena cava, inferior vena cava, right ventricle (for the atrial electrode), coronary sinus or pulmonary trunk. This position of the leads may be a consequence of dislodgement in the postoperative period or
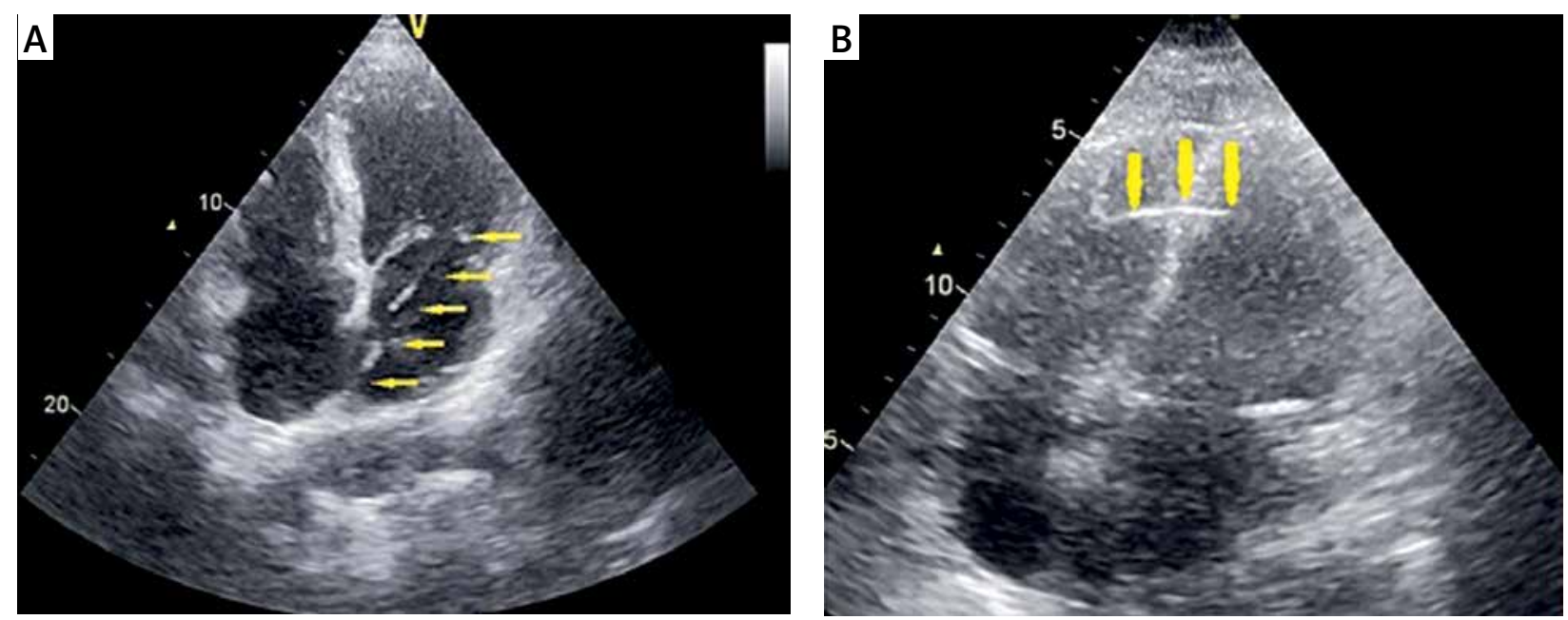

Figure 1. A - Mistaken implantation of the lead through the patent foramen ovale to the left ventricle, B - implantation of the lead to the left ventricle by the interventricular septum 
initially improper implantation. The effect may, but not necessarily, be a functional disturbance such as an increase in the pacing threshold, lead impedance changes, ineffective pacing, improper pacing for a given lead cavity, or impaired alertness due to low signal voltages [16, 17]. Under favorable circumstances, dislodgement may remain asymptomatic or nearly asymptomatic, resulting only in non-optimal sensing or stimulation parameters apart from improper lead location [15]. An example is the unintentional implantation of the right ventricular lead through the coronary sinus to the venous system of the heart [18]. In this way, effective left ventricular pacing is achieved from the direction of the epicardium, a situation similar to the one present in resynchronisation systems. However, the classic ventricular lead used for this purpose differs in physical properties, primarily the thickness and hence the rigidity of dedicated left-hand leads. This may threaten the perforation of the venous system with subsequent massive pericardial bleeding. The embedding of the lead into the thin-walled vein may also significantly hinder the attempt to remove such a system in the future. This type of complication usually remains asymptomatic, and diagnosis can be made by imaging studies such as echocardiography or chest X-ray, and primarily by a routine 12-lead ECG. In this situation, the morphology of the paced QRS satisfies the criteria for effective left ventricular stimulation: it is similar to a right bundle branch block (RBBB) in the precordial leads, with the dominant R wave in the V1 lead [19], but sometimes, if the lead is deep in the coronary sinus, near the apex, the ECG looks like the right ventricular pacing (LBBB).

Another cause of incorrect location of the lead may be its dislodgement. According to the literature and our own experience, the incidence of such complications ranges from $1.9 \%$ to $3.7 \%[8,20,21]$. Over many years, the leads used a passive fixation system, most often attached to the surrounding heart structures using dedicated insets, usually in the form of tendons. Such a lead construction allowed it to be fixed without the need for penetration into the myocardium, which theoretically reduced the chance of perforation of the heart cavity. However, the consequence of this solution is the possibility of losing the anchor of the lead in a moving organ such as the heart, thus creating the possibility of its displacement. This was especially the case in the early postoperative period, before the lead was attached to the endocardium, and the most affected by complications were atrial leads, due to the lack of rich trabeculation in the region of the right atrium. Widespread use of leads with an active fixation method has led to a significant decrease in dislodgement of the leads [22]. This complication usually needs surgical reintervention and reposition of the lead, but there are reported cases of successful percutaneous lead repositioning [23]. The percutaneous approach can be used for removal of broken parts of leads or guidewires [24].

\section{Early perforation}

Another early complication caused by improper location of the lead may be early perforation. This complication occurs in about $1.2 \%$ (0.07\% per year) of procedures $[8,20]$. It is a consequence of the dislocation or maneuvering of the lead (especially the ICD) at the implantation stage [22], but it also occurs as a complication of the use of transvenous temporary pacing [25] and its risk increases significantly with systemic steroid use, which significantly weakens the endurance of the myocardium [26, 27]. Other risk factors for perforation include female gender, low body mass index (BMI), advanced age, anticoagulant therapy, and active lead fixation $[28,29]$. Perforation of the free atrial or ventricular wall can lead to bleeding into the pericardium. In less severe cases bleeding tends to be self-limiting; in more severe cases it can lead to cardiac tamponade requiring pericardial drainage, blood transfusions, and sometimes even cardiac surgery [25].

\section{Hematoma of the generator pocket}

A hematoma of the generator pocket is one of the more common complications, observed in $1.4-3.2 \%$ patients $(0.4 \%$ per year) after implantation or pacemaker replacement [8, 20, 21]. Clinically, it may appear very different - from the small sizes of bloody infarcts, highlighting the edges of the pocket through the palpable outlines of the device to massive tumors located in the vicinity of the pocket, accompanied by an infarct occupying a large part of the chest, or even the entire body, which leads to a significant decline in the morphotic parameters of the blood, often requiring immediate intervention, including transfusion of blood products [30] (Figure 2). Patients usually complain about the pain of this area and the feeling of spreading. This complication may arise due to incomplete hemostasis or coagulation disorders, congenital or much more often iatrogenic, as a result of anticoagulation or antiplatelet treatment $[31,32]$. Of great importance in the prevention of this type of complication was the introduction of drugs from the novel oral anticoagulant (NOAC) group and the release of ESC guidelines which sanctioned uniformly carrying out anticoagulation treatment during the peri-implantation period, with a recommendation limiting bridging therapy to patients with prosthetic valves [33]. Thus, it significantly reduced the need for bridging therapy using low molecular weight heparins, which are considered risk factors for hematomas [29]. This is important due to the fact that the hematoma is one of the strongest risk factors of infectious complications in the long term [34].

\section{Pneumothorax and pleural hematoma}

Pneumothorax is most often a consequence of an attempt at venous access by puncture of the subclavian vein or its preparation, which results from its anatomical- 


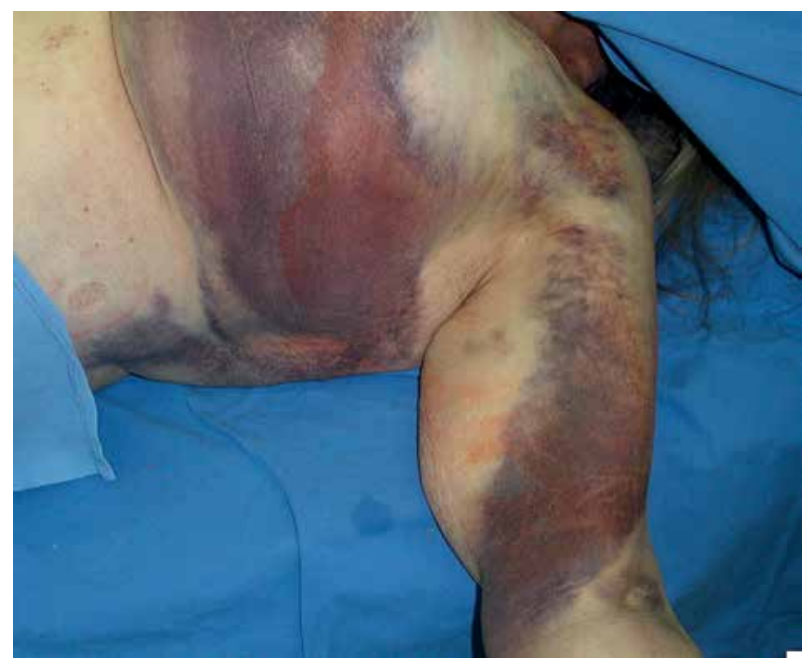

Figure 2. Extensive hematoma after implantation of pacing system

ly close location to the pleura [35]. Usually it is situated on the same side as the punctured vein, but there is also possible contralateral localization [36]. The incidence of this complication is estimated at $1.9 \%$ ( $0.3 \%$ per year) $[8,20]$. Clinical manifestations of pneumothorax may vary considerably - from the absence of symptoms to circulatory and respiratory insufficiency. Factors conducive to the development of such complications are CRT [26] or DDD implantation, chronic obstructive pulmonary disease, female sex and advanced age [37]. The best way to prevent this type of complication is to use venesection instead of a puncture of the vein. According to recent reports, e.g. Hadjis et al. [38], it is possible to implant CRT devices using the cephalic vein approach.

Pleural hematoma after implantation of the pacing system is described very rarely and may be due to perforation of the heart cavity [39].

\section{Early dysfunction of the lead}

Early dysfunction of the lead may be caused by an exit or entry block or a microdislodgement of the lead. The exit block is associated with an increase in the pacing threshold with a simultaneous moderate increase in lead resistance, which may lead to ineffective pacing [40, 41]. This phenomenon is very rare nowadays $(<0.1 \%)$ [8]. Entry block manifests itself as a decrease in the amplitude of the intracardiac potential, which may be accompanied by an increase in the threshold of pacing, usually without change in resistance. In both cases there is no dislocation of the lead and the cause is endocardial fibrosis in the lead tip region [40, 42]. Frequently a change of pacemaker parameters observed soon after implantation is a result of microdislodgement. In this situation failure to pace or sense is a consequence of minor movement of the lead tip [43]. Another reason for transient change pacing parameters is tissue injury associated with the operation. Also anti-arrhythmic drugs (propafenone), as well as systemic events such as anemia or electrolyte disturbances, especially hyperkalemia, can increase the pacing threshold, rendering the heart tissue unexcitable in extreme situations. Myocardial ischemia can cause the same effect. A heart attack involving the area directly adjacent to the lead can lead to permanent loss of pacing due to scar formation there. Electrical cardioversion can cause the same effect [41]. The difference between these states, however, is that in the case of a typical exit or entry block, progressive scarring around the tip of the lead is a response to the irritating effect of pacing pulses and is usually permanent and progressive. Commonly used for preventing this complication are steroid-releasing leads that should inhibit the inflammatory process in the apical region of the lead, and thus also the development of connective tissue in this area [40].

\section{Venous thrombosis}

Early venous thrombosis occurs in about $0.7 \%$ cases of implantation [8] and may be symptomatic or asymptomatic. Trauma caused by implantation of the pacing system, turbulent and slowed blood flow, and the irritating effect of the leads on the endothelium of the vein may cause a blood clot which results in impaired blood outflow from a particular area [40,42]. Depending on the location of thrombosis and the individual variability of the venous system, the symptoms, mainly in the form of pain and edema, may affect the upper limb on the side of the implanted device, but also the neck and face, and sometimes they may lead to the development of superior vena cava syndrome, with stagnation and congestion throughout the upper half of the body. Symptoms are sometimes poorly expressed or transient [44]. This may be a consequence of partial or complete recanalization or the development of hemodynamically effective collateral circulation, even if the organizing thrombus leads to permanent venous occlusion. Due to the generally poorly expressed symptoms of thrombosis, its diagnosis in the early postoperative period is quite rare. However, the recognized thrombosis is treated with anti-coagulant drugs, most commonly low molecular weight heparins, which will lead to the dissolution of the thrombus and finally to the restoration of venous system patency [44]. In addition to the cessation of symptoms, this may be significant in the future if leads are to be removed or the pacing system is expanded.

\section{Early and late infections}

\section{Local pocket infections and lead-related infective endocarditis (LRIE)}

The presence of a pacing system, as with any other type of implant, is associated with an increased risk of developing infectious complications. The overall incidence of cardiac device infections (CDI) ranged from $0.5 \%$ to $2.2 \%$ of patients [45]. These complications may 
be related to clinical factors (renal failure, diabetes mellitus, immunosuppression, steroid therapy, hematoma of the generator pocket, male gender) [40, 44-46] but also the type and complexity of the system (ICD, CRT-D/P, presence of inactive leads, and procedural factors such as type of procedure, generator replacement, number of leads, loops of the leads, intracardiac lead abrasion and fever up to $24 \mathrm{~h}$ prior to surgery, temporal transvenous pacing, surgical asepsis, a lack of antibiotic perioperative prophylaxis and reintervention - especially early replacement of equipment and operator experience [6, 40, 46, 47]. It is very important to prevent the development of infection including pre-operative systemic antimicrobial prophylaxis one hour prior to skin incision, surgical skin antisepsis and compliance with the discipline of the operating room. The antibiotic used for prophylaxis should have activity against the most common causative microorganism. The best evidence of benefit comes from a trial using cefazolin as the active agent, but trials of prophylaxis in cardiac device infections have not taken into account the long incubation period of many CDI and further randomized trials may be needed to determine the optimal agents $[45,48]$. In addition to systemic antibiotic prophylaxis, studies on the local use of antibacterial envelopes are ongoing. A current meta-analysis of five cohort studies demonstrated a significant beneficial effect of the TYRX antibiotic envelope (releasing minocycline and rifampin) for the prevention of CDI [49]. In turn, the results of a retrospective study evaluating the efficacy of the AIGISRx envelope, also releasing minocycline and rifampin, showed a higher rate of CDI in patients with this envelope [50]. The results of the Worldwide Randomized Antibiotic EnveloPe Infection PrevenTion Trial (WRAP-IT) are expected [51]. Another factor considered to contribute to the risk of infectious complications is operator experience. The analysis of previous studies has not confirmed the impact of the operator's experience on CDI development, but some studies demonstrated a higher overall complications rate in low volume centers [45, 52].

The most frequent pathogens are Staphylococcus epidermidis and Staphylococcus aureus [53, 54]. The pathogen may infect the pacing system already during implantation, and also during subsequent manipulation, through skin penetration of its constituents or via the bloodstream associated with passing bacteremia. The severity of symptoms depends on the virulence of the pathogen and the efficiency of the immune system of the patient. Local early infection of the pocket is manifested by the lack of healing of the wound, shedding of the edges of the wound, redness, excessive warmth or pain, the presence of purulent discharge or swelling of the pocket area. Sometimes, it leads to complete or partial emergence of the device. In the past, a popular form of therapy was local surgical treatment of the pocket, when all elements of the pacing system were left in the patient's body, which led to the suppression of the inflammatory process and a delay in proper management - transvenous lead extraction $[3,6]$.

Lead-related infective endocarditis can be created by the spread of infection by the continuity of the pocket along the course of the leads or it can originally occupy the leads and endocardium (blood infection). The clinical manifestation is less pronounced than with left-sided endocarditis. Symptoms can include recurrent sweats, weight loss, fever and subfeverish states of unknown origin, which are signs of repeated or chronic bacteremia, while cough, recurrent pneumonia, and dyspnea result from pulmonary embolism of bacterial material from the right heart $[3,6$, $46,47,55]$. In this group of patients it is harder to fulfill the classic Duke criteria necessary for the diagnosis of infective endocarditis, which is the reason for their suggested modification for LRIE and joining the major criteria for local pocket infection and septic pulmonary embolism [55-57]. Clinical practice often shows negative blood cultures as a result of empirically managed outpatient antibiotic therapy. The basic imaging technique in the diagnosis of LRIE is echocardiography, especially transesophageal echocardiography (TEE), due to better visualization of the leads (the proximal section of the superior vena cava), and also the possibility of a more accurate assessment of vegetations and the tricuspid valve $[6,55,57]$. In the case of a negative result of the transthoracic echocardiography (TTE) and TEE of a positive blood culture and continued suspicion of LRIE, intracardiac echocardiography and PET/CT using 18F-DG or radiolabeled leukocytes may be considered [6].

Endocarditis is a form of septic condition with all its possible consequences. In the case of LRIE, the presence of an implant in the form of a pacing system makes the appropriately isolated antibiotic treatment unsuitable. In this situation, whether the infection involves the endocardium, the pocket, or both, it is necessary to remove the entire implanted system. The treatments are usually performed by the transvenous method, currently even in the case of a large vegetation connected with leads $[3,6]$. Treatment supplements are antibiotics empirically effective against staphylococci (vancomycin, daptomycin). The recommended duration of use for LRIE is 4-6 weeks, including at least 2 weeks after removal, and should be extended to 4 weeks with positive blood cultures $>24 \mathrm{~h}$ after TLE. In the case of an isolated pocket infection the time of antibiotic therapy used is shorter (14 days) [6]. The problem is the unambiguous demarcation between the infection limited to the pocket and LRIE. The cultures obtained from the lead during TLE, although recommended, may have no differentiating power due to the possibility of their contamination during treatment while passing through the pocket.

\section{Late complications}

Decubitus of pocket and threatening decubitus

Pocket decubitus is most often produced by the superficial location of the generator. Predisposing factors 
are advanced age, external irritants and insufficient adipose tissue. Shallow location of the unit due to chronic skin irritations and the pressure exerted on it may lead to necrosis of the skin [58]. Until the moment the skin is tight and immovable, we recognize threatening decubitus (Figures $3 \mathrm{~A}, \mathrm{~B}$ ). The appearance of epidermal defects is associated with the diagnosis of decubitus, which is identical to infection of the pocket, because it is colonized by dermal bacterial flora (Figures 3 C, D). In the case of threatening decubitus the creation of a new pocket of the device in the deeper layers of the tissue, on the pectoralis major or below it, is recommended. Decubitus should be treated as a local infection of the pocket [58].

\section{Late perforation}

Late perforations are defined as occurring over a month after lead implantation $[3,58]$. Their actual incidence is difficult to estimate, and ranges from $1 \%$ to $14 \%$ of all late complications [3, 15]. Late perforations are asymptomatic or symptomatic, and often irregularities affect only electrical parameters, although they are often found to be normal despite obvious perforations in imaging studies [3]. Patients' stated symptoms are often
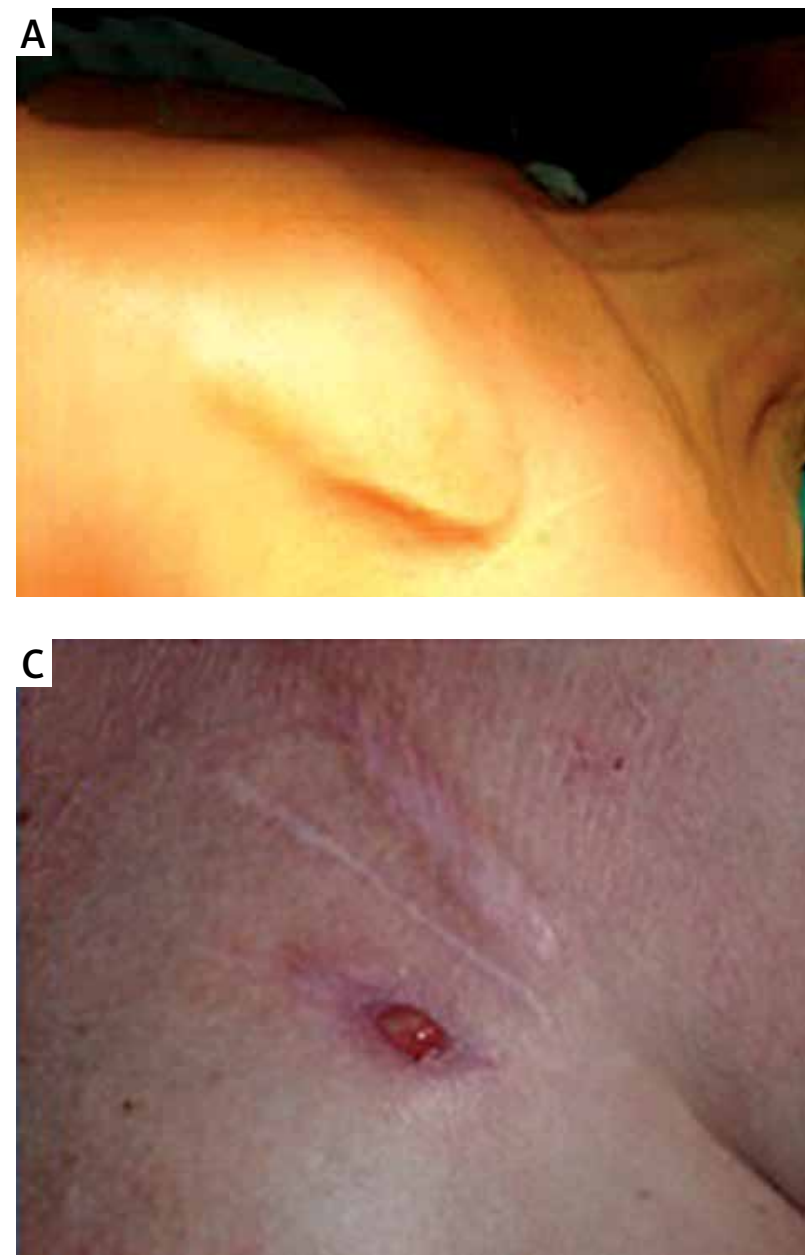

uncharacteristic, including chest pain, usually of a stabbing nature, periodic lack of breath, or involuntary muscle spasms within the chest, hiccups, dizziness, weakness or recurrent fainting $[3,59]$. Late perforations are the result of excessive stress on the lead, which, during any heart contraction, exerts its pressure on the muscle in a spearlike mechanism, which ultimately leads to the penetration of the lead by the heart muscle into the pericardium, neighboring heart chambers or surrounding structures. Factors that increase the risk of perforation include atrial leads, active fixated leads [58], defibrillation leads and leads implanted in the right ventricle, as well as female gender, advanced age and low BMI $<20 \mathrm{~kg} / \mathrm{m}^{2}$ [3]. No symptoms or low severity are due to the fact that most often they are microperforations, connected with penetration of only the tip of the lead beyond the contour of the heart. At the same time these are so-called dry perforations without the presence of fluid or with the form of a lens of fluid located near the tip of the lead (Figure 4). This is a consequence of the chronic nature of the complication and the presence of pericardial fatty tissue, which is capable of sealing the heart wall by overgrowing the tip of the lead [3]. Demonstrating lead dys-
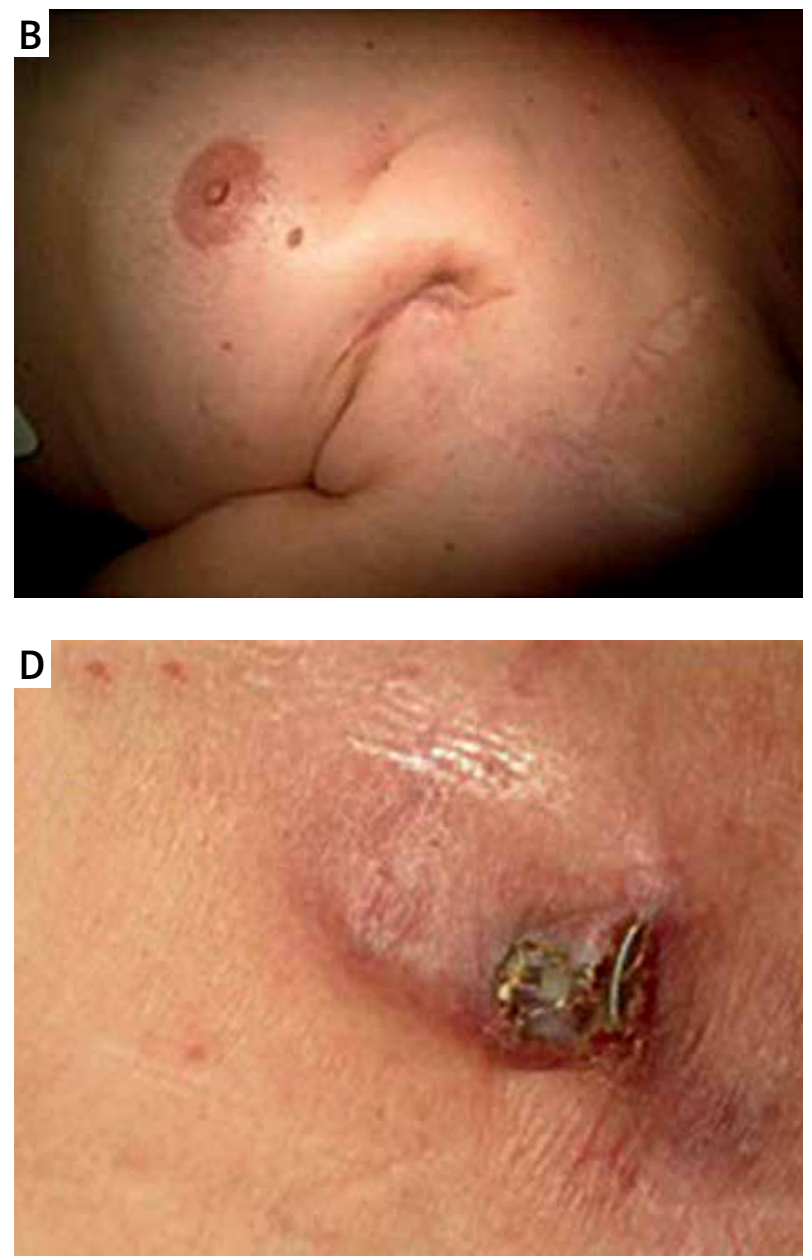

Figure 3. A, B - Threatening decubitus of the pocket, C, D - recurrent/chronic decubitus (pocket infection) 
function by changing electrical parameters is required for a diagnosis to be made. In the classical situation, the intra-ventricular voltage drops, the pacing thresholds rise, and the resistance of the lead may both increase and decrease in the long term $[3,59]$. The variable image of parameters results from the different nature of the tissue with which the electrode may come into contact - fat tissue, which has high resistance, or fluid, which is a good conductor. Confirmation of perforation can be demonstrated by means of echocardiography of the tip of the pericardium or even outside of the pericardium, although computed tomography (CT) is considered the gold standard $[3,58]$. Management of such cases is not explicitly determined and needs to be updated in the light of the new research findings. The 2009 HRS guidelines do not recommend TLE for atypical lead locations [4]. Clinical practice shows, however, that TLE is probably an effective and safe method of treatment. According to analysis of a large database (1536 patients undergoing TLE in the years 2006-2013 by A. Kutarski), among 211 patients with late perforations, procedural major complications related to perforations were observed in $3(1.4 \%)$ patients (data unpublished).

\section{Late-recognized lead dislodgement. \\ Late-identified incorrect lead location}

Both types of complications are a consequence of prior dislocation or initially improper implantation of the lead. The early detection of these abnormalities is possible based on ECG tracing and should be confirmed by imaging tests (RTG, fluoroscopy, echocardiography). However, in spite of the improper location of the lead, it is sometimes possible to provide effective pacing with its accepted parameters (usually an increase in the pacing threshold and a decrease in intracardiac potentials), which may lead to a lack of recognition of these complications for a long time. Only imaging studies, often performed for other reasons, indicate the improper location of the leads. Rules of the procedure are similar to those of early complications of this type, taking into account the time that has elapsed since implantation.

\section{Lead migration}

Lead migration is most often a consequence of loosening the strap that secures the lead or its originally insufficient anchorage [3]. This can result in excessive mobility and lead to the displacement of the excess lead or abandoned lead from the pocket to the heart chambers and loop formation within the atrium or ventricle, and sometimes even in the region of the pulmonary trunk (Figure 5) $[60,61]$. This can also lead to the loss of insulation or conduction by the rubbing of fragments of loops against each other or the rubbing of loops against neighboring heart structures [3]. As a consequence, pacing and sensing disorders are frequently encountered,

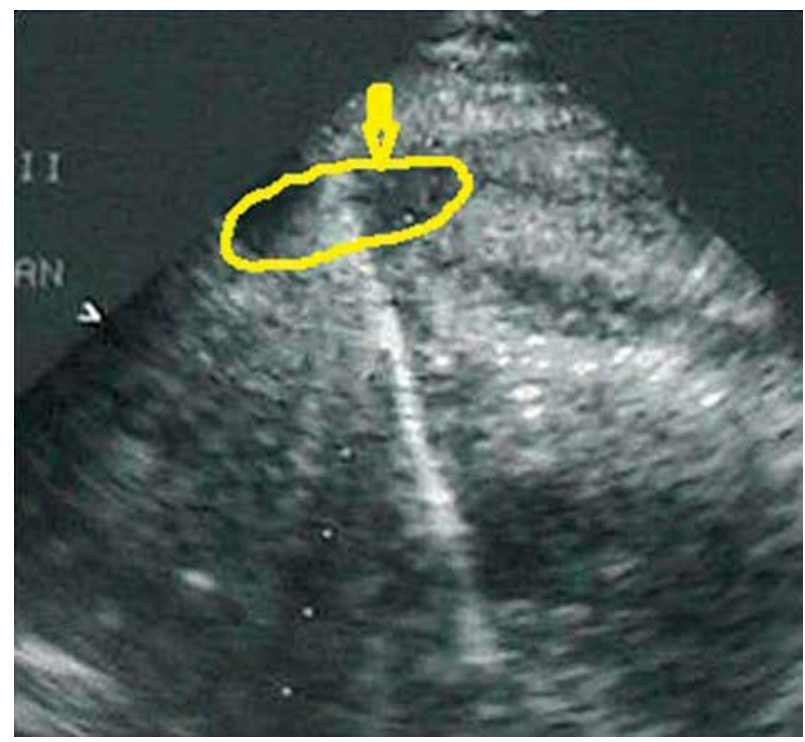

Figure 4. Dry perforation by the lead with the lens of fluid

and in the case of defibrillation leads inadequate ICD discharges are encountered in response to alleged ventricular tachyarrhythmias resulting from the cracking of the insulation and friction of the leads. Damaged leads, especially their sheaths, can also be the starting point for LRIE development. The damaged casing reveals thrombogenic material, and provides the bacteria with a conducive microenvironment, not only rich in nutrients, but also isolated from the patient's immune system and even protected against systemic antibiotics [3].

Another possible effect of lead loops is their irritating effect on the cardiac structures, which may provoke arrhythmias, including ventricular ones, sometimes in their more severe forms (VT or VF) [3]. Loose lead loops can also grow onto the endocardium, which significantly impairs their elimination. Lead growth into structures such as chordae tendineae and tricuspid valve muscles may lead to a change in the geometry of the subvalvular apparatus and the development of lead-dependent tricuspid dysfunction (LDTD) [3, 62]. Another mechanism of LDTD is the mechanical opening of the leaflets by the loop of the lead, which may prevent their proper coaptation [63].

Another example of a complication in which we observe lead migration is pacemaker twist (twiddler's syndrome or reel syndrome), which is an expression of the excessive mobility of the unit within the pocket. Such situations can occur in the case of a bulky pocket, as well as in pathologically obese patients or patients with advanced cognitive disorders. The complication arises due to the rotation of the unit within the pocket, which leads to multiple turns of the leads along their long axis (twiddler's syndrome) or transverse axis (reel syndrome) so that they resemble a spring as a consequence. Twiddler's 

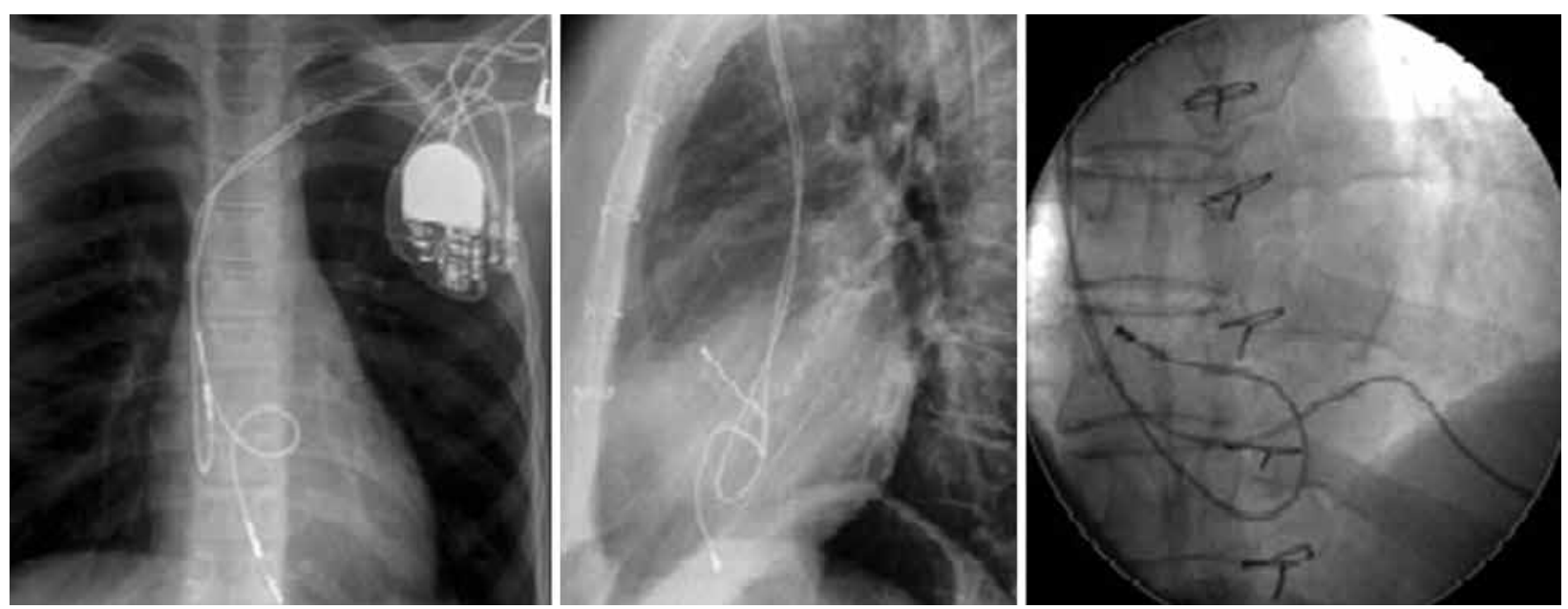

Figure 5. Loops of the leads

syndrome occurs later than reel syndrome (within a year after implantation) and is associated with worse complications than reel syndrome. Numerous stress points can lead to physical damage to the lead, abrasion and breakage of the conductor or displacement of the generator, and consequently to disturbances in the pacing. Reel syndrome develops earlier - within a month after implantation, and does not cause damage of the lead $[41,58,64,65]$.

\section{Lead fractures}

Lead fractures in distal observation are reported in $2.8 \%$ of cases [59]. Physical damage to the leads in their course through the venous system and consequently dysfunction can occur through a variety of mechanisms. The outer sheath, acting as insulation, the metal conductor of the lead core, or both at the same time can be destroyed. A broken conductor is the most common cause of lead dysfunction necessitating TLE [3]. Isolated insulation damage usually results from rubbing leads, their fragments or friction of the lead with surrounding cardiac structures, and may also be the result of a tootight gauze clamp [3]. The consequence is the "escape" of the current from the system, which can manifest as a sharp decrease in lead resistance, increased pacing thresholds, ineffective pacing, or premature battery exhaustion. There is also hyper-sensitivity of the device associated with the occurrence of artefacts in the form of cracks resulting from friction of the conductors themselves or other structures of the heart [37, 38]. In the case of high-energy systems, this may result in inadequate discharges due to VT/VF malfunction due to "cracks" in the ventricular channel and, in the case of PM, may lead to inhibition of pacing. Damaged cushions may also be LRIE's starting point [3]. In turn, damage to the conductor leads to a rapid increase in resistance, often preceded by fluctuations, which results in a decrease in the amount of energy delivered to the tissues and often results in a lack of response to the pacing impulse. At the same time the voltages of the intracardiac potentials decrease, which may result in the device being under-sensitive. In addition, the breaking wire can be a source of artefacts with all the possible consequences mentioned above (inadequate ICD discharge, inhibition of pacing) [3, 37, 38].

A classic example of lead fracture is the crushing syndrome. It occurs in the space between the clavicle and the first rib as a consequence of the too medial puncturing of the subclavian vein [38]. Due to the anatomical proximity of these structures, the conductor can be crushed and destroyed (both the conductor and isolation). In order to avoid this complication, a more lateral puncture of the subclavian vein should be done, or alternatively, insertion of the leads by the cephalic vein can be performed [66].

\section{Chronic venous occlusion}

Although chronic venous insufficiency and acute venous thrombosis may be similar in terms of clinical symptoms, these two states must be distinguished from each other due to the varied nature of the underlying lesions. The estimated incidence of chronic venous occlusion ranges from $20 \%$ to as high as $75 \%[3,67]$. Acute thrombosis can, but does not necessarily, lead to chronic occlusion. Venous occlusion is caused by an inflammatory response to the presence of leads or their implantation $[3,67]$. The presence of the lead itself slows blood flow and irritates the vein walls because of its natural mobility in all planes. Consequently, there is a tissue reaction that eventually leads to significant narrowing or loss of patency. The particularly exposed place is the division of vessels. The identified factors contributing to the development of occlusion are the number of previous treatments, the development of the pacing system, the number of leads and the sum of their size [3, 40, 66]. Chronic venous occlusion may produce symptoms similar to acute thrombosis, assuming a gradual build- 
up of thrombosis. Even asymptomatic obstruction can be a problem if one needs to perform dialysis fistulae, vascular ports, a central line, an extension of system, replacement of leads or TLE procedures with different indications. Because of the nature of the lesions that are of connective tissue, pharmacodynamic effects, including those of anticoagulants, should not be expected. In case of a need to regain vascular access, already implanted leads should be used, which after the introduction of the guidewire can lead to the introduction of new leads or the treatment of a stenting vein.

\section{Lead-dependent tricuspid dysfunction (LDTD)}

Implantation of the pacing systems may lead to the appearance or worsening of pre-existing tricuspid regurgitation. It is twice as common in patients with implanted devices as in the general population. Significant deterioration of the function of the tricuspid valve after implantation is observed in $7.2-39 \%$ of patients $[68,69]$. The most common cause of LDTD is the presence of a lead or leads passing through the annulus of the valve deliberately as in the case of ventricular leads or unintended leads as with dislocated atrial leads or additional lead loops, as discussed in detail in the section on migration of leads. The chance for significant tricuspid dysfunction to develop increases with the number of leads, including abandoned ones, or their loops passing through the valve $[40,63,68]$. The mechanism itself in which regurgitation occurs may vary. The most obvious is to support the leaflet using the lead, eventually leading to the fusion of the leaflets to the lead. Another mechanism is through the presence of a loop-caused problem with coaptation of the leaflets. Also, the interaction between the lead and the subvalvular apparatus may prevent it from closing. The ingrowing of the chordae tendineae or papillary muscle to the lead results in limitation of its mobility and ultimately impairs the tightness of the right venous outlet. Regurgitation due to mechanical damage to the valve or subvalvular apparatus is varied in nature, and can lead to rupture of the leaflet, perforation, or a break in the chordae tendineae. Finally, the mechanism of the expansion of the valve annulus itself must be mentioned [68]. As long as the LDTD is a consequence of mobility disorders of the leaflets without damaging them, the chance of improving the function of the valve after removal of the lead is high, yet mechanical damage to the valve causes that after the lead is removed the regurgitation will not disappear and may even intensify. To improve the function of the valve, it is advisable to remove the lead by the transvenous method with careful dissolution of all points of contact between the lead and the surrounding tissue in such a way that the treatment itself does not contribute to its destruction. Otherwise, cardiac surgery may be necessary with either repair or replacement of the valve [68]. The LDTD prevention depends on such a course of the lead through the valve that it does not support any of the lobes and runs optimally around the annulus through the spoil [68].

\section{Conclusions}

Optimal treatment of patients with electrotherapy complications requires proper diagnosis. First and foremost, it is important to remember that complications, especially late ones, are becoming more and more frequent due to the increasing population of people with many years of pacing systems. Apart from the local evaluation of the pacemaker pocket and the control of the device parameters (sometimes also observation of their long-term trends may be necessary), imaging studies play an important role in determining the nature of the complications and the potential for dealing with them. In the case of chronic complications of electrotherapy the basis of their treatment is transvenous lead extraction, whose role and safety in these indications have been confirmed.

\section{Conflict of interest}

The authors declare no conflict of interest.

\section{References}

1. Aquilina O. A brief history of cardiac pacing. Images Paediatr Cardiol 2006; 8: 17-81.

2. Świątecka G. History of cardiac pacing in Poland. W Dobrym Rytmie 2013; 3: 4-8.

3. Polewczyk A, Kutarski A, Tomaszewski A, et al. Late complications of electrotherapy - a clinical analysis of indications for transvenous removal of endocardial leads: a single centre experience. Kardiol Pol 2013; 71: 366-72.

4. Wilkoff BL, Love CJ, Byrd CL, et al. Transvenous lead extraction: Heart Rhythm Society expert consensus on facilities, training, indications, and patient management: this document was endorsed by the American Heart Association (AHA). Heart Rhythm 2009; 6: 1085-104.

5. Tayebjee MH, Joy ER, Sandoe JA. Can implantable cardiac electronic device infections be defined as 'early' or 'late' based on the cause of infection? J Med Microbiol 2013; 62: 1215-9.

6. Habib G, Lancellotti P, Antunes MJ, et al. ESC Guidelines for the management of infective endocarditis: The Task Force for the Management of Infective Endocarditis of the European Society of Cardiology (ESC). Eur Heart J 2015; 36: 3075-128.

7. Wazni O, Wilkoff BL. Considerations for cardiac device lead extraction. Nat Rev Cardiol 2016; 13: 221-9.

8. Pakarinen S, Oikarinen L, Toivonen L. Short-term implantationrelated complications of cardiac rhythm management device therapy: a retrospective single-centre 1-year survey. Europace 2010; 12: 103-8.

9. Van Gelder BM, Bracke FA, Oto A, et al. Diagnosis and management of inadvertently placed pacing and ICD leads in the left ventricle: a multicenter experience and review of the literature. Pacing Clin Electrophysiol 2000; 23: 877-83.

10. Nordbeck $P$, Langenfeld $H$, Krein $A$, et al. Pacing lead inserted via the subclavian artery caused acute coronary syndrome, Herzschrittmacherther Elektrophysiol 2008; 19: 188-92. 
11. Lepore V, Pizzarelli G, Dernevik L. Inadvertent transarterial pacemaker insertion: an unusual complication. Pacing Clin Electrophysiol 1987; 10: 951-4.

12. Shulz N, Püschel K, Turk EE. Fatal complications of pacemaker and implantable cardioverter-defibrillator implantation: medical malpractice? Interact CardioVasc Thorac Surg 2009; 8: 444-8.

13. Alozie A, Westphal B, Yerebakan C, et al. Transient ischaemic attack due to the lead of an implantable defibrillator in the left heart. Interact Cardiovasc Thorac Surg 2012; 14: 128-30.

14. Kutarski A, Pietura R, Tomaszewski A, et al. Transvenous extraction of a five year-old ventricular lead inadvertently implanted in the left ventricle. Kardiol Pol 2011; 69: 625-9.

15. Wynn GJ, Weston C, Cooper RJ, et al. Inadvertent left ventricular pacing through a patent foramen ovale: identification, management and implications for postpacemaker implantation checks. BMJ Case Rep 2013; 27: 1-3.

16. Fuertes B, Toquero J, Arroyo-Espliguero R, et al. Pacemaker lead displacement: mechanisms and management. Indian Pacing Electrophysiol J 2003; 3: 231-8.

17. Rydlewska A, Suślik W, Małecka B. Spontaneous pacemaker distal lead tip migration resulting in lack of ventricular stimulation. Folia Cardiol 2014; 9: 179-81.

18. Shettigar UR, Loungani RR, Smith CA. Inadvertent permanent ventricular pacing from the coronary vein: an electrocardiographic, roentgenographic, and echocardiographic assessment. Clin Cardiol 1989; 12: 267-74.

19. Hao Y, Li Y, Liao D, et al. A comparative analysis of the effectiveness of active versus passive atrial lead fixation in Chinese patients with cardiac implantable electrical devices: a long term, retrospective, observational, single-center study. Curr Med Res Opin 2017; 33: 573-8.

20. Palmisano P, Accogli M, Zaccaria M, et al. Rate, causes, and impact on patient outcome of implantable device complications requiring surgical revision: large population survey from two centres in Italy. Europace 2013; 15: 531-40.

21. Piątek $九$, Polewczyk A, Kurzawski J, et al. Analysis of the incidence and causes of repeated surgical interventions in patients with early complications electrotherapy - 1 center experience from the period 2012-2015. Przegl Lekarski 2016; 73: 378-81.

22. Khan MN, Joseph G, Khaykin Y, et al. Delayed lead perforation: a disturbing trend. Pacing Clin Electrophysiol 2005; 28: 251-3.

23. Ozel E, Osztheimer I, Ozturk A, et al. Percutaneous right atrial pacemaker lead repositioning using a regular deflectable ablation catheter. Adv Interv Cardiol 2016; 12: 183-4.

24. Engel AM, Machejek J, Ząbek A, et al. CRT-D implantation: one procedure, two problems. Adv Interv Cardiol 2016; 12: 282-4.

25. Banaszewski M, Stepinska J. Right heart perforation by pacemaker leads. Arch Med Sci 2012; 8: 11-3.

26. Lin YS, Hung SP, Chen PR, et al. Risk factors influencing complications of cardiac implantable electronic device implantation: infection, pneumothorax and heart perforation: a nationwide population-based cohort study. Medicine (Baltimore) 2014; 93 e213.

27. Mahapatra S, Bybee KA, Bunch TJ, et al. Incidence and predictors of cardiac perforation after permanent pacemaker placement. Heart Rhythm 2005; 2: 907-91.

28. Hermanides RS, Ottervanger JP, Elvan A, et al. Life-threatening perforation of a defibrillation lead. Neth Heart J 2009; 17: 113-4.

29. Sterliński M, Przybylski A, Maciąg A, et al. Subacute cardiac perforations associated with active fixation leeds. Europace 2009; 11: 206-12.
30. De Sensi F, Miracapillo G, Cresti A, et al. Pocket hematoma: a call for definition. Pacing Clin Electrophysiol 2015; 38: 909-13.

31. Wiegand UKH, LeJeune D, Boguschewski F, et al. Pocket hematoma after pacemaker or implantable cardioverter defibrillator surgery: influence of patient morbidity, operation strategy, and perioperative antiplatelet/anticoagulation therapy. Chest 2004; 126: 1177-86.

32. Tompkins C, Cheng A, Dalal D, et al. Dual antiplatelet therapy and heparin "bridging" significantly increase the risk of bleeding complications after pacemaker or implantable cardioverter defibrillator device implantation. J Am Coll Cardiol 2010; 55: 2376-82.

33. Kirchhof P, Benussi S, Kotecha D, et al. 2016 ESC Guidelines for the management of atrial fibrillation developed in collaboration with EACTS. Eur Heart J 2016; 37: 2893-962.

34. Raad D, Irani J, AkI EG, et al. Implantable electrophysiologic cardiac device infections: a risk factor analysis. Eur J Clin Microbiol Infect Dis 2012; 31: 3015-21.

35. Res JCJ, de Priester JA, van Lier AA, et al. Pneumothorax resulting from subclavian puncture: a complication of permanent pacemaker lead implantation. Neth Heart J 2004; 12: 101-5.

36. Hardzina M, Ząbek A, Boczar K, et al. Contralateral pneumothorax after cardiac pacemaker implantation. Postep Kardiol Interw 2015; 11: 347-8.

37. Kotsakou M, Kioumis I, Lazaridis G, et al. Pacemaker insertion. Ann Transl Med 2015; 3: 42.

38. Hadjis A, Proietti R, Essebag V. Implantation of cardiac resynchronization therapy devices using three leads by cephalic vein dissection approach. Europace 2017; 19: 1514-20.

39. Nichols J, Berger N, Praveen J. Subacute right ventricle perforation by pacemaker lead presenting with left hemothorax and shock. Case Rep Cardiol 2015; 2015: 983930.

40. Madhavan M, Mulpuru SK, McLeod CJ, et al. Cardiac pacemakers: function, troubleshooting, and management. J Am Coll Cardiol 2017; 69: 189-210.

41. Barold SS, Stroobandt RX, Sinnaeve AF. Stymulatory serca. Przewodnik ilustrowany. Urban \& Partner, Wrocław 2006.

42. Łebek-Szatańska A, Przychodzeń A, Dąbrowski M. Subclavian vein thrombosis after pacemaker implantation - case report. Acta Angiol 2013; 19: 93-8.

43. Kikuchi K, Abe H, Nagatomo T, et al. A likely mechanism of pacing failure with high impedence small area electrodes. PACE 2003; 26: 1541-3.

44. Sohail MR, Uslan DZ, Khan AH, et al. Risk factor analysis of permanent pacemaker infection. Clin Infect Dis 2007; 45: 166-73.

45. Sandoe JA, Barlow G, Chambers JB, et al. Guidelines for the diagnosis, prevention and management of implantable cardiac electronic device infection. J Antimicrob Chemother 2015; 70: 325-59.

46. Polewczyk A, Jacheć W, Tomaszewski A, et al. Lead-related infective endocarditis: factors influencing the formation of large vegetations. Europace 2017; 19: 1022-30.

47. Polewczyk A, Jacheć W, Polewczyk AM, et al. Infectious complications in patients with cardiac implantable electronic devices - risk factors, prevention and prognosis. Pol Arch Intern Med 2017; 127: 597-607.

48. de Oliveira JC, Martinelli M, Nishioka SA, et al. Efficacy of antibiotic prophylaxis before the implantation of pacemakers and cardioverter defibrillators: results of a large, prospective, randomized, double-blinded, placebo-controlled trial. Circ Arrhythm Electrophysiol 2009; 2: 29-34. 
49. Ali S, Kanjwal Y, Bruhl SR, et al. A meta-analysis of antibacterial envelope use in prevention of cardiovascular implantable electronic device infection. Ther Adv Infect Dis 2017; 4: 75-82.

50. Hassoun A, Thottacherry ED, Raja M, et al. Retrospective comparative analysis of cardiovascular implantable electronic device infections with and without the use of antibacterial envelopes. J Hosp Infect 2017; 95: 286-91.

51. Tarakji KG, Mittal S, Kennergren C, et al. Worldwide randomized antibiotic EnveloPe Infection PrevenTion Trial (WRAP-IT). Am Heart J 2016; 180: 12-21.

52. Freeman JV, Wang Y, Curtis JP, et al. The relation between hospital procedure volume and complications of cardioverter-defibrillator implantation from the implantable cardioverter-defibrillator registry. J Am Coll Cardiol 2010; 56: 1133-9.

53. Villamil Cl, Rodriguez FM, Van den Eynde CA, et al. Permanent transvenous pacemaker infections: an analysis of 59 cases. Eur J Intern Med 2007; 18: 484-8.

54. Bongiorni MG, Tascini C, Tagliaferri E, et al. Microbiology of cardiac implantable electronic device infections. Europace 2012; 14: 1334-9.

55. Klug D, Lacroix D, Savoye C, et al. Systemic infection related to endocarditis on pacemaker leads: clinical presentation and management. Circulation 1997; 95: 2098-107.

56. Sohail MR, Uslan DZ, Khan AH, et al. Infective endocarditis complicating permanent pacemaker and implantable cardioverter-defibrillator infection. Mayo Clin Proc 2008; 83: 46-53.

57. Vilacosta I, Sarria C, San Roman JA, et al. Usefulness of transesophageal echocardiography for diagnosis of infected transvenous permanent pacemakers. Circulation 1994; 89: 2684-7.

58. Gul EE, Kayrak M. Common pacemaker problems: lead and pocket complications. In: Modern Pacemakers - Present and Future. Das MM. InTech, 2011; 299-318. Available at: http://www.intechopen.com/books/modern-pacemakers-present-and-future/ common-pacemaker-problems-lead-and-pocket-complications

59. Refaat MM, Hashash JG, Shalaby AA. Late perforation by cardiac implantable electronic device leads: clinical presentation, diagnostic clues, and management. Clin Cardiol 2010; 33: 466-75.

60. Polewczyk M, Jacheć W, Polewczyk AM, et al. Leads dislodged into the pulmonary vascular bed in patients with cardiac implantable electronic devices. Adv Interv Cardiol 2016; 12: 348-54.

61. Polewczyk M, Polewczyk AM, Kutarski A, et al. Proximal end of 15-year-old ventricular electrode penetrating pulmonary tissue - a source of infection and a challenge for transvenous lead extraction. Postep Kardiol Interw 2015; 11: 248-9.

62. Al-Bawardy R, Krishnaswamy A, Bhargava M, et al. Tricuspid regurgitation in patients with pacemakers and implantable cardiac defibrillators: a comprehensive review. Clin Cardiol 2013; 36: 249-54.

63. Polewczyk A, Kutarski A, Tomaszewski A, et al. Lead dependent tricuspid dysfunction: analysis of the mechanism and management in patients referred for transvenous lead extraction. Cardiol J 2013; 20: 402-10.

64. Munawa M, Munawar DL, Basalamah F, Pambudi J. Reel syndrome: a variant form of Twiddler's syndrome. J Arrhythmia 2011; 27: 338-42.

65. Majewski J, Lelakowski J, Haberka K, et al. Trwałość stymulacji dwujamowej w długotrwałej obserwacji. Folia Cardiol 2002; 9: 567-72.

66. Aizawa Y, Negishi M, Kashimura S, et al. Predictive factors of lead failure in patients implanted with cardiac devices. Int J Cardiol 2015; 199: 277-81.
67. Abu-El-Haija B, Bhave PD, Campbell DN, et al. Venous stenosis after transvenous lead placement: a study of outcomes and risk factors in 212 consecutive patients. J Am Heart Assoc 2015; 4: e001878.

68. Seo Y, Ishizu T, Nakajima H, et al. Clinical utility of 3-dimensional echocardiography in the evaluation of tricuspid regurgitation caused by pacemaker leads. Circ J 2008; 72: 1465-70.

69. Al-Mohaissen MA, Chan KL. Prevalence and mechanism of tricuspid regurgitation following implantation of endocardial leads for pacemaker or cardioverter-defibrillator. J Am Soc Echocardiogr 2012; 25: 245-52. 